\title{
CUANDO LA COMUNIDAD GUÍA LA ACCIÓN: HACIA UNA EVALUACIÓN COMUNITARIA ALTERNATIVA
}

\author{
Hélène Laperrière \\ Universidade de Ottawa, Ottawa, Canadá \\ Ricardo Zúñiga \\ Universidade de Montreal, Montreal, Canadá
}

RESUMEN: Cuando los modelos habituales de evaluación de programas son utilizados irreflexivamente en la evaluación de la acción comunitaria, ellos pueden privilegiar una visión de control social que olvida y oculta la realidad vivida por los grupos evaluados. 1. Los instrumentos de esta desposesión de derechos pueden ser ideologías exportadas como productos universales. 2. El peso del acto concreto es crucial para afirmar la realidad que se pretende modificar. 3. Las retóricas científicas ocultan la realidad vivida por los sectores sociales con menos poder. 4. Las responsabilidades éticas y políticas de la evaluación comunitaria exigen que los planes de intervención sean conocidos, comprendidos y asumidos por todos los que deberán vivir con las consecuencias del cambio intentado.

PALABRAS CLAVES: Evaluación comunitaria; participación; acción social.

\section{WHEN COMMUNITY GUIDES ACTION: \\ UNTIL AN ALTERNATIVE COMMUNITY EVALUATION}

ABSTRACT: When habitual models of program evaluation are used unreflectively for the evaluation of community action programs, they can focus exclusively on administrative accountability. 1. This limitation of participation can be due to the latent ideologies exported as universal products. 2. Understanding the concrete acts performed underlines the concrete reality of the situation that the project aims to modify. 3. Scientific discourses can obscure the life experiences of the relatively powerless social sectors. 4. Ethical and political responsibilities demand that intervention plans be known, shared and accepted by all those who will have to live with the intended and unintended consequences of the attempted social change.

KEYWORDS: Community evaluation; participation; social action.

La psicología social comunitaria ha desarrollado una gran variedad de tópicos, elegidos como prioritarios por criterios raramente explícitos. Uno de los dejados en el olvido relativo es el de la evaluación comunitaria. Situada en la tensión entre exigencias externas de tipo presupuestario y vivencias colectivas que buscan su voz para expresar su sentido, su proyecto y sus realizaciones, su estrategia comunicativa puede variar desde la aceptación - pasiva o aparente-de los encuadres externos hasta expresiones de afirmación de autonomía que pueden confrontar su dependencia real de los financiamientos que no controla. En estas notas, quisiéramos desarrollar los fundamentos para la lectura que los participantes directos de una acción colectiva hacen o pudieran hacer de ella, para comprenderla como acto transformador y como reivindicación.

\section{Las ideologías científicas y sus instrumento: cambio social, desarrollo, participación, empowerment}

Las ciencias sociales norteamericanas y luego europeas han hecho del «cambio social» un valor abstracto, aparentemente consensual: parece que «es bueno que las cosas cambien». Algunas experiencias dolorosas, como en la antropología (Casanova, 2002; Stavenhagen, 1981, 1971/ 1992) y en las ciencias políticas (Horowitz, 1967/1974) han mostrado la ingenuidad del supuesto de virtud intrín- seca del cambio no especificado. ¿De qué cambio estamos hablando? ¿Cambio de qué situación a cuál otra? ¿Cambio positivo o bueno según quiénes? ¿Con qué costos previstos y asumidos? Cuando el cambio parece ser evolutivo, sin costos aparentes, hay poco revuelo; cuando es «radical» (Zúñiga, 1975), el cambio desgarra una comunidad en sus fundamentos mismos, y no puede sino marcar toda acción con los rasgos de la elección dolorosa entre la preservación del orden o su derrocamiento. Como Horowitz lo denunció tan fundadamente, el cambio social es «progreso y desarrollo» 0 «insurgencia y terrorismo» según la relación del proceso de producción científica a sus contextos políticos. Esta conciencia política es central en la constitución de la investigación social.

Los obstáculos a la liberación de los pueblos, las comunidades y las personas se dan en todos los campos de la actividad social. Entre ellos, la salud es especialmente importante por su apariencia objetiva y científicamente neutra, que fundamenta su poder. La perspectiva del trabajo se desarrolla a partir de la premisa que toda innovación en los métodos de evaluación está ligada a opciones metodológicas que permanecen en la penumbra de lo implícito. Las bases del análisis son la construcción colectiva de un útil de evaluación de los grupos de la Coalición de organismos comunitarios de lucha contra el SIDA (COCQSida) en el Québec (Jalbert, Pinault, Renaud \& Zúñiga, 
1997;Zúñiga,Luly \& COCQ-Sida, 2005) y las actividades de prevención de las MTS, el VIH y el SIDA en la región amazónica del Brasil (Laperrière, 2004, 2006).

Nuestro trabajo es una reflexión teórica imbricada en las formas de práctica en las que se realizó, en los diálogos que la originaron. Nuestra posición es que la evaluación de la acción comunitaria, realizada en una perspectiva de investigación-acción participativa, es fértil tanto para la comprensión de la acción, que es el objeto de reflexión para tomar conciencia de las opciones metodológicas que esta perspectiva metodológica permite identificar con claridad.

\section{Los costos políticos de una metodología truncada}

La promoción de la salud es muchas veces una forma de colonización en dos niveles: (a) internacional, desde centros de poder que se presentan como "mundiales" para proyectarse hacia periferias receptoras, y (b) interna, en que la salud es una forma particularmente eficaz de poder político de los centros nacionales sobre las comunidades locales. Los instrumentos de la colonización encontrados han sido: (a) Una concepción teórica aparentemente abstracta, que marginaliza la realidad concreta de la acción local y (b) una utilización ideológica des términos como "participación", "empowerment” y "concientización" todos términos útiles y aún necesarios, pero sólo a condición que se utilicen con una conciencia vigilante de las connotaciones ideológicas que pueden desvirtuarlos, limitándolos a una esfera de diálogo interpersonal, evitando el cuestionamiento de sus concepciones implícitas de la acción sociopolítica.

Toda evaluación de la acción social se realiza entre la idea que la guía y la acción transformadora que la realiza. La idea que guía la acción contiene los valores, las aspiraciones y las esperanzas que dinamizan la acción; la realidad de la acción concreta confronta la idea generosa y optimista con los hechos que reflejan el difícil proceso de cambiar una realidad humana. Si la visión era producir un cambio, una transformación, ¿Qué muestra que ha habido un cambio, que la idea ha logrado arraigarse, transformando la realidad? Esta tensión gatilla en el evaluador la presión ética de la toma de conciencia de sus propios valores. ¿Qué le permite ver el vaso no sólo lleno hasta la mitad, sino de expresar esta constatación como medio lleno o medio vacío? La evaluación es el encuentro del mundo de las ideas con el de las evidencias, y su expresión no podrá forzar el lenguaje de uno sobre el otro...

Una lectura realista de los resultados de una acción puede verlos como el resultado de una pugna entre la idea preconcebida de un cambio y la resistencia que le opone una situación preexistente, que no percibe necesariamente el cambio propuesto como uno que se integre sin rupturas a un mundo que les es ya conocido y que no cuestione vínculos humanos y sociales que son pilares de su estructura comunitaria. La eficacia de un proyecto de prevención puede ser evaluada por otros indicadores que se añadan a las estadísticas o a las observaciones externas, reconociendo que la incorporación de qué sea importante para los usuarios y los actores directamente implicados enriquece la mirada evaluativa. Estos actores hablan de «valores» al enunciar los medios que favorecerán la eficacia de estos proyectos: «Todo punto de vista es la visión desde un punto específico. Para comprender cómo alguien dice, es necesario saber cómo son sus ojos y cuál es su visión de mundo. Lo que hace que toda lectura sea una relectura» (Boff, 2003, p. 9). Brandão (2002, p. 151), uno de los militantes más activos de la educación popular brasileña, describe la investigación participativa como un modo de aprender a escribir la historia a partir de la propia y acerca de los movimientos populares. Al referirnos a Boff (2003) y sobre todo a Freire (1980), es importante, sin embargo, subrayar que estos autores son actualmente víctimas de un «endulzamiento ideológico», principalmente en el Canadá y Estados Unidos. Este endulzamiento ideológico busca reducir su aporte a una humanización compatible con una visión desarrollista, centrada en el desarrollo individual. Se oculta, así, el fundamento esencial de sus posiciones como el rechazo de un orden social injusto, la incitación a una conciencia socialmente activa, revolucionaria en el sentido profundo del término.

La experiencia brasileña ha sido analizada como la triple refracción de un prisma: la irrupción de imprevistos sociales y de la verticalidad de las relaciones entre los actores (Laperrière \& Zúñiga, 2006), y la consideración de la peligrosidad para los participantes de contextos de violencia física y simbólica. Los proyectos de prevención permiten insertarse en la realidad estructurante de las culturas organizacionales locales, que incluyen aspectos que el investigador no puede marginalizar, como la historicidad de estas organizaciones que define la visión de los participantes. El concepto de verticalidad busca el subrayar que, fuera de los canales jerárquicos explícitos y implícitos, están los poderes reconocidos informalmente, que en la región del estudio incluyen los narcotraficantes, los propietarios de los bares en los que se establece la prostitución, las bandas callejeras y los políticos locales, con los que el proyecto de investigación no puede negociar como lo hace con los pares «visibles y reconocidos» del sistema oficial de salud (Laperrière, Benzaken \& Zúñiga, 2004). En el caso de la evaluación brasileña, la situación de investigación era una que estaba inmersa en un contexto sociopolítico de peligrosidad reconocida. Los actores sociosanitarios, policiales, gubernamentales y delictuales tejían una red de relaciones complejas que la investigación tradicional prefiere ignorar, pero que la vida colectiva refleja en su cotidianeidad: el no hablar de pandillas, del narcotráfico y de las tensiones en torno a la acción de la policía 
y de los administradores políticos no bastaba para aislar la investigación de su contexto. Las consecuencias concretas de este realismo son dobles. Por una parte, hay que responder al desafío de defender las realidades locales, tal como son vividas, y no sólo ideas abstractas de progreso y de desarrollo. Por otra, es necesario tener claro el problema de las colonizaciones como mecanismos que pueden estar basados en buenas intenciones, pero que siempre son importaciones con efectos insospechados de destrucción de configuraciones culturales y valorales.

En un trabajo previo (Laperrière \& Zúñiga, 2006), hemos explicado en detalle que, para nosotros, la noción de verticalidad es más amplia que la de jerarquías formales de control. Los proyectos interactúan con las agencias que los fundan y con la imagen pública, sobre todo política, que los identifica; los comités de evaluación y de ética no dan órdenes ni prohibiciones, pero expresan opiniones y recomendaciones difíciles de ignorar. Los datos de nuestros estudios muestran que esta influencia descendente no tiene una continuidad formal, ni es una estructura unificada, pero que marca una dominación descendiente de la información que afecta las decisiones que se toman. La acción se apoya en datos en movimiento, muchas veces ambiguos y desconcertantes, difíciles de formalizar y de cuantificar. Es el producto de una flexibilidad y de una adaptación continua a circunstancias cambiantes. Ella debe reformular la verticalidad como una interdependencia reconocida y respetada, aún dentro de una lógica de subordinación.

El segundo caso que nos sirve de material empírico de reflexión y de construcción teórica es el de las prácticas evaluativas formales de organismos comunitarios de lucha contra el SIDA en el Québec. La situación confrontada era la de cómo dar cuenta del trabajo realizado a la fuente de financiamiento gubernamental. Los programas gubernamentales subvencionados funcionan con formularios rígidos, genéricos, centrados en la producción de estadísticas de productividad del organismo como organización formal - la voz de la Dirección General del organismo dando cuenta de la utilización de fondos en función de los resultados alcanzados.

Los grupos comunitarios tiene muy claro que confrontan una triple tarea para mostrar su acción y demostrar su contribución: desarrollar una voz autónoma (la misión del organismo como integración de los programas subvencionados), alimentar internamente la acción y enriquecer el discurso de justificación. El desafío es hacer de ellas tareas que sean vistas por el conjunto de sus miembros como modalidades de búsqueda y de expresión de la razón de ser misma de los grupos. Ello implica una tarea antes no confrontada de buscar una palabra que corresponda a las tareas reales, que capte las actividades en su riqueza concreta antes de decidir qué aspectos conservar en el discurso oficial.
La tensión entre conversaciones informales internas al grupo y los discursos formalizados destinados a responder a exigencias externas no es sólo una oposición de actores. El proyecto ha estado siempre fomentado por los organismos de financiamiento, que lo han visto como un aporte. El primer documento producido ha sido recomendado para el uso de todos los organismos de la Coalición, y las agencias gubernamentales han dejado en claro que esperan que sea utilizado en todas las evaluaciones e informes de progreso. Pero el trabajo no ha sido fácil. La perspectiva metodológica se ha desarrollado en interacción con la experiencia de grupos de trabajo, consultas a grupos específicos, y reuniones de intervinientes.

El proceso de elaboración de útiles evaluativos se abocó a dos desafíos. Por una parte, la definición implícita de la evaluación como investigación evocaba fantasmas de lenguaje abstruso, de estadísticas que provenían de indicadores impuestos. Por otra, los objetivos debían sobrepasar la idea que eran necesariamente descendentes, que eran sólo la formulación de las exigencias externas del financiamiento, para incorporar el que eran también la expresión de la dinámica de acción colectiva. Tampoco era necesariamente cierto que el no alcanzar objetivos predeterminados era siempre la culpa de los intervinientes, sino que podía deberse a la falta de realismo de los objetivos tal como formulados por las cúpulas administrativas, determinados a priori y en función de criterios externos. La consideración del contexto y la adaptación progresiva del proyecto a sus circunstancias concretas de actualización no eran defectos, sino virtudes de la acción. Los trabajos de los proyectos de preparación de útiles de evaluación fueron concebidos y realizados como ocasiones de coconstrucción de significaciones de las experiencias vividas a diario, y que debían reflejar y expresar la identidad colectiva del grupo, que era una conciencia de su acción:

Cuando hablamos hoy en día de identidad, espontáneamente la formulamos como una pregunta: «QQuién soy?». Nuestra respuesta constituye una respuesta de qué es lo que es esencialmente importante para nosotros. Saber quién soy implica que yo sepa dónde me sitúo. Mi identidad se define en los compromisos y las identificaciones que determinan en encuadre o el horizonte en el cual puedo juzgar caso a caso lo que está bien, lo que convendría hacer, qué es lo que acepto y a qué me opongo. En otros términos, mi identidad es el horizonte con referencia al que puedo tomar posiciones. (Taylor, 1989, p. 27).

Las dos experiencias de terreno, ambas de varios años de duración, tenían una intencionalidad abstracta común (la prevención del Sida), pero con formas muy distintas de acción. En ambas, el trabajo fue concebido y realizado tratando de maximizar la participación de los participantes en todas las etapas de las evaluaciones, desde la definición de metas y de medio, a la construcción de estrategias de 
recolección y de análisis de información y a la redacción de los informes. En ambas se generó un sector de mediación, de participantes que actuaron como agentes multiplicadores y de contacto. En ambas fue posible romper la barrera de la palabra escrita, al menos al reducir las desconfianzas hacia ella. Una medida de éxito de los proyectos es el grado en que la implicación de estos mediadores los hizo apropiarse de la experiencia, llevando su compromiso con ella mucho más allá que una simple obligación. Otra, igualmente provechosa, fue la generación de un discurso que los investigadores siempre sometieron a la consideración de esos colaboradores, que definieron su participación no sólo en las tareas a realizar sino también en la influencia que tenían en el producto final, que debía responder a sus criterios de claridad y de utilidad.

\section{La acción pensada \\ y la reafirmación del acto concreto}

La relación de la palabra a la acción es central a la definición de la función de la evaluación como escrito. Las palabras preceden, determinan y reemplazan la acción concreta y pueden inhibir la palabra que nombra la acción transformadora. Los conflictos epistémicos de la evaluación tienen siempre el mismo origen: se considera el espacio pensado como si fuera una confirmación del mundo real. "Así nace algo como un segundo mundo teórico junto al mundo percibido y concebido. ¿Y quién decide si esas dos visiones del mundo se contradicen?" (Riedl, 1989, p. 70). La respuesta de Mendel es clara: el concepto de acción ha absorbido el acto como fenómeno, hasta tal punto que ambos parecen haberse fusionado, haber llegado a ser intercambiables - pero siempre en favor del primer término. El acto es así concebido como un reflejo pálido de la idea. No incluye ni la resistencia a la intención que el sujeto intenta imprimir sobre el mundo, ni tampoco la interacción de éste con el mundo, que lo modifica como sujeto mientras cree modificar el mundo (Mendel, 1998). Su perspectiva crítica ha sido central en nuestro análisis.

Mendel (1998) nos invitaba a tomar conciencia que el poder no era sólo el ejercido por unos sobre otros, sino también como el poder que individuos y colectividades ejercen sobre sus propias acciones, y es éste el que el evaluador debe asegurarse de incluir en su evaluación de la reapropiación que los participantes pueden hacer de su propia acción (ver Figura 1). En sus términos, la acción como concepto ha absorbido el acto como fenómeno, hasta tal punto que ambos parecen haberse fusionado, haber llegado a ser intercambiables - pero siempre en favor del primer término. El acto así concebido es una distorsión por omisión de la acción. No incluye ni la resistencia a la intención que el sujeto intenta imprimir sobre el mundo, ni tampoco la interacción de éste con el mundo, que lo modifica como sujeto mientras cree modificar el mundo (Mendel, 1998).

Una evaluación limitada a los objetivos se limita a la verificación de la relación entre 1 y 3 entre un objetivo propuesto y los resultados alcanzados, que debieran corresponderse estrechamente. Una evaluación integral de impacto sigue la ruta $1-5-3$, y acepta que el paso por el acto lleva a producir un resultado que no es necesariamente el previsto. El acto es, al fin y al cabo, el encuentro difícil de una idea preconcebida con una realidad previamente estructurada que la resiste (Laperrière, 2006).

El peso del acto es crucial para anclar la evaluación en un contacto real con la realidad que pretende modificar (ver Figura 2). La previsión o el «pre-acto» no debieran limitarse a buscar el resultado esperado, empobreciéndolo al observarlo sólo a través del filtro de los indicadores pre-establecidos. Cuando los conceptos aceptan integrarse al mundo de la acción, el camino de la acción incluye la totalidad de los actos realizados, así que el cómo son incorporados o resistidos. El resultado es, así, una novedad imprevisible, la historia de una interacción del mundo de las ideas del sujeto con el de su realización en una realidad que le es externa, que lo precede y que le opone la fuerza de lo ya existente.

La ruptura posible entre las conceptualizaciones importadas y las acciones y los conceptos instrumentales en la acción directa (Laperrière, 2006) ilustra dos lógicas de evaluación diferentes (Campbell, 1984; Mendel, 1998; Scriven, 1972, 2004). Ellas fundan la distinción de Scriven

\begin{tabular}{|c|c|c|c|}
\cline { 2 - 3 } $\begin{array}{c}\text { La acción imaginada, } \\
\text { y el resultado esperado - } \\
\text { como IDEAS }\end{array}$ & $\begin{array}{c}\text { La idea de la acción prevista: } \\
\text { EL PRE-ACTO }\end{array}$ & $\begin{array}{c}\mathbf{2} \\
\text { La acción propuesta tal } \\
\text { como "debiera" expresar } \\
\text { la previsión }\end{array}$ & $\begin{array}{c}\text { El resultado } \\
\text { esperado previsto: } \\
\text { EL POST-ACTO }\end{array}$ \\
\cline { 2 - 4 } $\begin{array}{c}\text { La conciencia } \\
\text { en durante la acción }\end{array}$ & $\begin{array}{c}\text { Conocer los actores } \\
\text { implicados y cómo } \\
\text { comprenden la tarea común }\end{array}$ & $\begin{array}{c}\text { Comprender la acción } \\
\text { como interacción entre el } \\
\text { proyecto y la realidad: } \\
\text { EL ACTO }\end{array}$ & $\begin{array}{c}\text { Comprender los resultados } \\
\text { como el resultado del } \\
\text { encuentro de un posible } \\
\text { con una realidad concreta }\end{array}$ \\
\hline
\end{tabular}

Figura 1. La acción pensada y la conciencia del acto (Laperrière, 2006; versión modificada por Laperrière \& Zúñiga, 2006). 
LA ACCION COMO UNA IDEA DE LO POSIBLE -

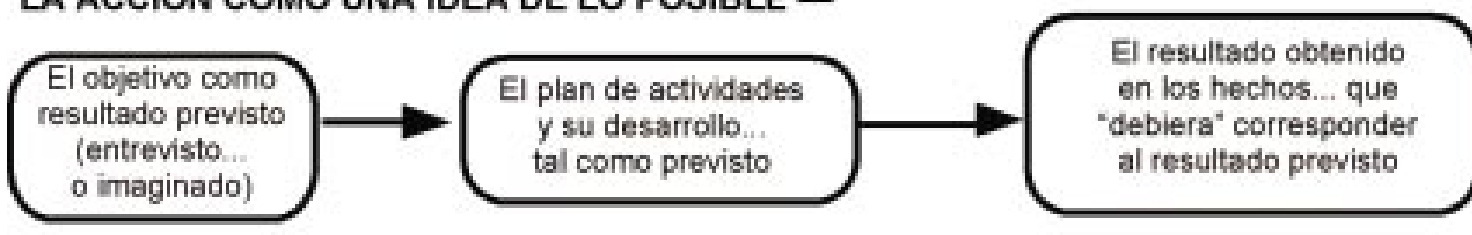

\section{... Y LA ACCION COMO PRODUCTORA \\ DE UNA NUEVA REALIDAD - \\ PARCIALMENTE IMPREVISIBLE}

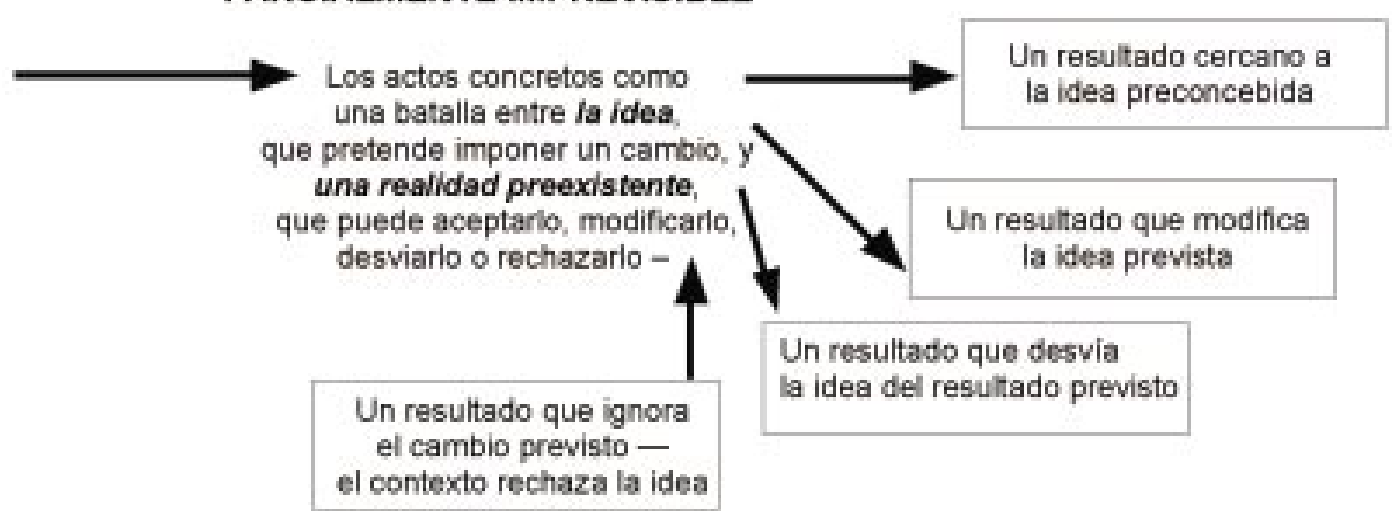

Figura 2. De la idea a la acción transformadora.

(1972) entre las lógicas de la "evaluación centrada en los objetivos", orientada hacia la verificación de los resultados esperados por los administradores del programa, y una "evaluación sin objetivos". Esta, que evidentemente no implica la carencia de objetivos del programa evaluado sino la ausencia de presupuestos en la observación evaluativa inicial, propone que la evaluación comience observando la situación de funcionamiento del programa y los cambios que aparecen como efectos o consecuencias de su actividad, sin cerrarse a observar sólo lo que corresponda al objetivo contractual tal como decidido por los administradores del programa y aceptado por el evaluador.

Las palabras que expresan intenciones y acciones son más que palabras. Ellas son actos transformadores, creadores de realidades nuevas. El trabajo de los filósofos del lenguaje (Austin, 1982; Flores, 1994; Maturana \& Varela, 1984; Searle, 1986) ha mostrado la palabra como diferente tanto de la idea como del acto material, como una variable intermediaria del acto transformador: ella es un «acto de habla». El señalar y el nombrar determinan las acciones sociales. Cuando la persona de color es un negro; cuando la trabajadora del sexo es una puta; cuando el niño viviendo un conflicto es un desadaptado o un deficiente y cuando el inmigrante es un sudaca, sus vidas han sido transformadas - empobrecidas, marginalizadas, menoscabadas - por una palabra. Que nadie les diga a ellos que las palabras no construyen realidades que vehiculan una eficacia mortífera.

También son actos de compromiso - el nombrar implica al actor que nombra. Los autores citados insisten en diferenciar claramente la idea de la palabra, que es idea, pero que es también reflejo de una interacción y forma de acción interactiva. Para ellos, las palabras son «conversaciones para la acción». «Las conversaciones para la acción son aquéllas mediante las cuales logramos que las cosas se hagan... una conversación para la acción, en su forma más sencilla, es una petición y una promesa de cumplirla» (Flores, 1994, p. 44). La evaluación es un ejemplo claro que exige un doble compromiso: hacer explícitos los valores y los criterios que fundamentan el juicio de valor que guía la evaluación, y asegurar la veracidad y la calidad de la información que es aportada. Maturana agrega una segunda nota en el resumen de su posición como los aforismos claves de su libro: «Todo hacer es conocer» $\mathrm{y}$ «Todo lo dicho es dicho por alguien» (Maturana \& Varela, 1984, p. 13). Las ideas que llevan a implicarse en el mundo concreto son ocasiones de progreso del conocer, y ese conocer nunca es impersonal: siempre es alguien que conoce, desde su historia personal, su posición social y sus compromisos valóricos y sociales. Es un «alguien» que busca respuestas a sus preguntas personales, la satisfacción de sus intereses. En situaciones de divergencia de intereses sociales, la evaluación escrita puede ser la palabra del fiscal acusador o del abogado defensor - pero difícilmente puede pretender ser la del juez que pueda clamar su neutralidad distante y desarraigada. Una evaluación alternativa deberá considerar la totalidad concreta del impacto de la acción, incorporando los cambios imprevistos del proyecto concebido en términos de la idea esperada.

La evaluación es la construcción de un actor social responsable que, justamente porque es responsable, busca 
Laperrière, H.; Zúñiga, R. "Cuando la comunidad guia la acción: hacia una evaluación comunitaria alternativa”

la eficacia en los cambios que juzga necesarios. Ver una realidad, juzgarla a través de los valores que definen una perspectiva, y actuar para acercarla a una realización más plena son los momentos de una acción social de transformación, que se fija objetivos, pero que los comprende como contextualizados, arraigados en una situación específica y en los intereses y tomas de posiciones de todos los que están implicados o afectados por los cambios propuestos. La evolución cualitativa puede contribuir, identificando estos actores e intereses y asegurándose que el proyecto evaluado y la evaluación no ignoren u olviden todo lo que pueda explicar el resultado específico obtenido por la acción.

\section{4. Ética y política de la evaluación comunitaria: historia colectiva y el sujeto-actor}

Las perspectivas futuras subrayan la convergencia de objetividad y subjetividades en la evaluación comunitaria como potencial de recuperación de la historia colectiva. Nuestra reflexión es el resultado de la relación entre nuestras convicciones, que sitúan toda investigación científica como implicando necesariamente consecuencias para las acciones prácticas - consecuencias que van mucho más allá de la teoría científica que las justifica y que pretende inmunizarlas de todo «contagio» con las realidades concretas que viven los participantes, muchas veces «sujetos» en distintos sentidos sutiles. En un intercambio en la investigación en la región amazónica, un participante exclamó: « Ahora comprendo qué es la evaluación! ¡Estamos escribiendo nuestra historia!». El proceso de la investigación participativa crea conciencia histórica - conciencia del mundo que se ha compartido, de las opciones personales con las que cada miembro del grupo contribuye a la construcción de una acción que es colectiva, pero que sigue siendo la expresión de la historia personal de cada participante.

La historia colectiva de los pueblos latinoamericanos es también una historia entre grupos, con diferencias culturales y sobre todo de poder. Demasiadas veces, ellas están encubiertas por discursos patrióticos que son los de las clases dirigentes. Creemos que toda reflexión sobre los métodos de evaluación está íntimamente ligada a opciones metodológicas que son necesariamente políticas (Zúñiga \& Laperrière, 2006). Nuestro trabajo en un esfuerzo de mostrar cómo, bajo las retóricas científicas, políticas y económicas, se oculta une realidad de personas con menos poder que viven en carne propia las ideas de cambio que se pretende imponerles en nombre de teorías científicas. La meta de una acción social - incluso en el área de la salud: es hacer emerger la historia colectiva de los grupos y de los pueblos que buscan su autonomía y su voz política y que muchas veces pagan el costo de sus convicciones con sus vidas. Este alto costo, asumido sobre todo por los más vulnerables, es el de que la comunidad gane su derecho a guiar su propio actuar.

\section{Referencias}

Austin, J. L. (1982). Cómo hacer cosas con palabras. Palabras y acciones. Barcelona, España: Paidós.

Boff, L. (2003). A águia e a galinha. Uma metáfora da condição humana (40. ed.). Petrópolis, RJ: Vozes.

Brandão, C. R. (2002). A educaçãgo popular na escola cidada. Petrópolis, RJ: Vozes.

Campbell, D. T. (1984). Can we be scientific in applied social science? In E. S. Overman (Ed.), Methodology and epistemology for social science (pp. 315-333). Chicago: The University of Chicago Press.

Casanova, P. G. (2002). Exploração, colonialismo e luta pela democracia na América Latina. Petrópolis, RJ: Vozes.

Flores, F. (1994). Creando organizaciones para el futuro. Santiago de Chile: Dolmen.

Freire, P. (1980). Conscientização: Teoria e prática da libertação. Uma introdução ao pensamento de Paulo Freire (3. ed.). São Paulo, SP: Morães.

Horowitz, I. L. (1974). The rise and fall of Project Camelot: Studies in the relationship between social science and practical politics. Cambridge, MA: The M.I.T. Press. (Original publicado em 1967)

Jalbert, Y., Pinault, L., Renaud, G., \& Zúñiga, R. (1997). Epsilon. Guide d'auto-évaluation des organismes communautaires. Montreal, Canadá: Coalition des organismes de lutte contre le Sida (COCQ-Sida).

Laperrière, H. (2004). L'évaluation de l'action préventive en contexte d'imprévisibilité. Les enjeux d'un projet de prévention des MTS/ VIH/Sida par les pairs, Amazonas, Brasil. Dissertação de Mestrado não-publicada, Faculdade das Ciências de Enfermagem, Universidade de Montreal, Canadá.

Laperrière, H. (2006, sept.). La investigación evaluativa de los contextos sociopolítico y sociocultural. Los desafíos metodológicos de la urgencia de las acciones sociosanitarias en regiones alejadas. Forum Qualitative Sozialforschung / Forum: Qualitative Social Research [Electronic version], 7(4), Art. 6. Retirado em 17 dez. 2006, de http://www.qualitative-research.net/fqs-texte/4-06/064-6-s.htm

Laperrière, H., Benzaken, A. S., \& Zúñiga, R. (2004, oct.). Les déterminants sociopolitiques dans l'action conjointe à paliers multiples. Les interlocuteurs dans un projet de prévention des MTS/VIH/ Sida. Trabalho apresentado no $2^{\text {nd }}$ International Conference on Local and Regional Health Programmes, Quebec, Canadá.

Laperrière, H., \& Zúñiga, R. (2006). Sociopolitical determinants of anAIDS Prevention Program: Multiple actors and vertical relationships of control and influence. Policy, Politics \& Nursing Practice, $7(2), 1-11$

Maturana, R. H., \& Varela, G. F. (1984). De máquinas y seres vivos. Autopoiesis: la organización de lo vivo. Santiago de Chile: Editorial Universitaria.

Mendel, G. (1998). L'acte est une aventure. Du sujet métaphysique au sujet de l'actepouvoir. Paris: La découverte.

Riedl, R. (1989). Las consecuencias del pensamiento radical. In P. Watzlawick (Ed.), La realidad inventada. ¿Cómo sabemos lo que creemos saber? (pp. 62-81). Barcelona, España: Gedisa.

Searle, J. R. (1986). Actos de habla. Ensayo de filosofía del lenguaje (2. ed.). Madrid, España: Cátedra.

Scriven, M. (1972). Pros and cons of goal-free evaluation. Evaluation Comment, 3(4).

Scriven, M. (2004). Reflections. In M. C. Alkin. Evaluation roots. Tracing theorists'views and influences (pp. 183-195). Thousand Oaks, CA: Sage. 
Stavenhagen, R. (1981). Seven fallacies about Latin America. In Between underdevelopment and revolution. A latin american perspective (pp. 1-20). New Delhi, India: Abhinav.

Stavenhagen, R. (1992). Cómo descolonizar las ciencias sociales. In M. C. Salazar(Ed.), La investigación-ación participativa. Inicios y desarrollos (pp. 37-64). Buenos Aires, Argentina: Humanitas. (Original publicado em 1971)

Taylor, C. (1989). Sources of the self: The making of the modern identity. Cambridge, MA: Harvard University Press.

Zúñiga, R. (1975). The experimenting society and radical social reform. The role of the social scientist in Chile's Unidad Popular Experience. American Psychologist, 99-115. [Tradução de El papel del psicólogo social en América Latina, reproduzido de I. MartínBaró (Ed.), 1985, Problemas de Psicología Social en América Latina, San Salvador, El Salvador: UCA.].

Zúñiga, R. (2004). La evaluación en la acción social. Autonomías y solidaridades. Retirado de http://homepage.mac.com/ricardo. b.zuniga

Zúñiga, R., Luly, M.-H., \& Coalition des organismes de lutte contre le Sida. (2005). Savoir-faire et savoir-dire. Un guide d'évaluation communautaire. Montreal, Canadá: COCQ-Sida. Retirado de http://www.cocqsida.com/

Zúñiga, R., \& Laperrière, H. (2006). A avaliação comunitária: Conflitos verticais e ambigüedades metodológicas. In F. Mercado \& M. L. Bosi (Eds.), Avaliação de programas e serviços de saúde: Enfoques emergentes (pp. 118-144). Petrópolis, RJ: Vozes.

Hélène Laperrière é Mestra em ciências de enfermagem, cursando atualmente o doutorado em Saúde Pública da Faculdade de Medicina e o MicroPrograma de nível doutorado do Programa Analyse et Évaluation des Interventions en Santé (AnÉis), Universidade de Montreal, Canadá. Atuou ao longo de seis anos com o movimento de educação popular no Brasil, o que orientou sua reinserção em bairros periféricos durante a concretização de pesquisas-ação participativas e avaliativas. É professora na Escola de

Enfermagem, Universidade de Ottawa, Canadá. Seus interesses atuais giram em torno de uma prática comunitária de enfermagem, a avaliação dos grupos comunitários e a educação popular em sáude. Endereço para correspondência: Universidade de Ottawa, Escola de Enfermagem, 451, Smyth, Ottawa (Ontario), K1H8M5, Canadá.

klaperri@uottawa.ca

Ricardo Zúñiga é Doutor em Psicologia Social Harvard University. Sua produção intelectual teve início no Instituto de Sociologia da Universidade Católica do Chile, na área de Psicología Social. Com o golpe miliar, continuou seu trabalho na City

University of New Cork. Atualmente é profesor de Serviço Social na Universidade de Montreal, Canadá. Seu trabalho tem se concentrado na profissionalização das ciencias sociais e nos desafios de desenvolver modelos abertos de produção e difusão de conhecimentos bem como na temática da avaliação, sobretudo no enfoque qualitativo, em países latinoamericanos, principalmente no Chile.

ricardo.zuniga@umontreal.ca

\section{Cuando la comunidad guia la acción: hacia una evaluación comunitaria alternativa}

Hélène Laperrière \& Ricardo Zúñiga

Recebido: 22/12/2006

$1^{\text {a }}$ revisão: $17 / 06 / 2007$

Aceite final: 3/08/2007 